\title{
PRODUCCIÓN Y CALIDAD DEL FORRAJE DE VARIEDADES DE AVENA EN FUNCIÓN DEL SISTEMA DE SIEMBRA Y DE LA ETAPA DE MADUREZ AL CORTE
}

\section{YIELD AND QUALITY OF FORAGE VARIETIES OF OATS ACCORDING TO PLANTING SYSTEM AND MATURITY STAGE AT CUTTING}

\author{
Sergio Ramírez-Ordóñes ${ }^{1 \star}$, David Domínguez-Díaz², José J. Salmerón-Zamora ${ }^{3}$, \\ Guillermo Villalobos-Villalobos ${ }^{2}$ y Juan A. Ortega-Gutiérrez ${ }^{2}$
}

\footnotetext{
${ }^{1}$ Dpto. de Zootecnia, Universidad del Papaloapan. Cd. Universitaria, Av. Ferrocarril s/n. 68400, Loma Bonita, Oax. ${ }^{2}$ Facultad de Zootecnia y Ecología, Universidad Autónoma de Chihuahua. Km 1 Perif. Francisco R. Almada. 31031, Chihuahua, Chih. ${ }^{3}$ Facultad de Ciencias Agrotecnológicas, Universidad Autónoma de Chihuahua. Av. Presa de la Amistad No. 31510. 31510, Cd. Cuauhtémoc, Chih.

*Autor para correspondencia (sramirez@unpa.edu.mx; sramirez_28@hotmail.com)
}

\section{RESUMEN}

El forraje de avena (Avena sativa L.) se usa extensamente en los sistemas de alimentación pecuaria en el noroeste del Estado de Chihuahua, México. Se evaluó el efecto de la variedad, sistema de siembra y estado de madurez al corte sobre el rendimiento y composición química del forraje de avena, en siete variedades sembradas en surco con contras y en plano sin surco, en condiciones de temporal (secano) en cinco localidades del noroeste de esa entidad. La cosecha se efectuó en tres etapas fenológicas: embuche (EMB), grano masoso (MAS) y madurez fisiológica del grano (MF). Se midió el rendimiento de materia seca (MS) en $\mathrm{kg} \mathrm{ha}^{-1}$, la composición química del heno (\%), y se estimó la materia seca digestible (MSD, \%) y la energía neta de lactancia $\left(\mathrm{EN}_{\mathrm{L}}\right.$, Mcal $\left.\mathrm{kg}^{-1}\right)$. Los tratamientos se distribuyeron en un arreglo experimental de parcelas sub-sub-divididas en un diseño de bloques completos al azar, en el que localidad fue la repetición, la parcela grande fue el sistema de siembra, la mediana el estado de madurez al corte, y la parcela chica la variedad. El sistema de siembra no afectó al rendimiento $(P=0.20)$ ni a la composición química $(P>0.05)$ del heno. A medida que la etapa de corte fue más cercana a MF el rendimiento de MS $(\mathrm{P}<0.01)$ se incrementó linealmente $\left(2247,3120\right.$ y $4475 \mathrm{~kg} \mathrm{ha}^{-1}$ para EMB, MAS y MF, respectivamente), y la proteína cruda disminuyó en forma cuadrática $(P<\mathbf{0 . 0 1})$. En proteína cruda hubo efecto de la interacción de variedad $x$ madurez al corte $(P<0.01)$. A medida que la cosecha fue más cercana a MF las fracciones de fibra disminuyeron ( $P$ $<0.05)$, e inversamente MSD y $\mathrm{EN}_{\mathrm{L}}$ aumentaron linealmente $(\mathrm{P}<0.05)$. La composición química del heno de avena mejoró al acercarse a la madurez fisiológica del grano debido a la disminución en su contenido de fibra, sin presentar diferencias importantes entre variedades.

Palabras clave: Avena sativa, heno, composición química, etapa de madurez, sistema de siembra.

\section{SUMMARY}

Oat forage (Avena sativa L.) is widely used in livestock feeding systems at the northwestern region of Chihuahua State, México. The effect of variety, planting system, and maturity stage at cutting on yield and chemical composition of oat forage were evaluated on seven varieties planted under rainfed conditions, in furrow diking and plane surfaces, in five locations at the northwestern region of Chihuahua state. Harvest was done at three crop growth stages: boot (BS), dough grain (DS) and physiological maturity of grain (PMS). Yield in kg $\mathrm{ha}^{-1}$ of hay dry matter (DM) and its chemical composition (\%) were measured; digestible dry matter (DDM, \%) and net energy for lactation (NEL, Mcal $\mathrm{kg}^{-1}$ ) were estimated. Treatments were distributed into a sub-sub-split experimental arrangement in a completely randomized blocks design; locations were the replications, main plots were planting systems, medium size plots were stages of maturity at cutting, and large plots were varieties. Planting system did not affect hay dry matter yield $(P=0.20)$ or chemical composition $(P>0.05)$. As the cutting stage approached PMS, DM yield linearly $(P<0.05)$ increased $(2247,3120$ and $4475 \mathrm{~kg} \mathrm{ha}^{-1}$ for BS, DS and PMS, respectively), while crude protein decreased nonlinearly $(P<\mathbf{0 . 0 5})$. A significant effect of the maturity $x$ variety interaction $(P<0.01)$ on crude protein was detected. As harvest approached PMS, fiber fractions decreased $(\mathrm{P}<0.05)$, while DDM and ENL increased linearly $(P<0.05)$. Oat hay chemical composition improved towards maturity of grain due to a decrease in fiber, without significant differences among varieties.

Index words: Avena sativa, hay, chemical composition, maturity stage, planting system.

\section{INTRODUCCIÓN}

La avena (Avena sativa L.) es una importante planta productora de grano en varios países, que también se utiliza como forraje para la alimentación de animales en pastoreo, heno o ensilado. Esta gramínea produce forraje de buena calidad cuando otros cultivos forrajeros de mejor calidad son escasos. En México la superficie sembrada con avena en riego y temporal (secano) se incrementó de 311218 ha en 1990 a 942823 en 2011. En este mismo año se establecieron 885728 ha de avena en condiciones de temporal, de las cuales $93.7 \%$ se destinaron para forraje y $6.3 \%$ para grano (SIACON, 2011).

En el noroeste del Estado de Chihuahua la avena se cultiva principalmente en condiciones de temporal; su siembra representa $76 \%$ de la superficie establecida en México y 69 $\%$ de la superficie sembrada se destina para la producción de forraje. De la producción de esta especie en Chihuahua, 70 \% se emplea en la alimentación pecuaria, $25 \%$ para consumo humano y $5 \%$ para semilla (Ávila y Salmerón, 1999). Esta 
gramínea es la base del sistema de producción regional de leche (en la comunidad menonita, el forraje de avena representa $71 \%$ del costo total de alimentación del ganado lechero), y también se usa en los sistemas extensivos de bovinos productores de carne (Ávila et al., 2006).

Tradicionalmente, en Chihuahua la cosecha del forraje de avena se hace en la etapa de madurez fisiológica del grano, con rendimientos de 2.0 a $3.0 \mathrm{t}$ de forraje seco ha ${ }^{-1}$ (Ávila $e t$ al., 2006), con un contenido de proteína cruda (PC) menor de $10.5 \%$, el de fibra detergente neutro (FDN) mayor a 61.4 $\%$, y el de energía neta de lactancia $\left(\mathrm{EN}_{\mathrm{L}}\right)$ de $1.63 \mathrm{Mcal} \mathrm{kg}^{-1}$ de MS (Salmerón et al., 2003). Cuando se cosecha en etapa de embuche o grano masoso, la PC puede ser de 21 a $12 \%$ y la FDN de 44 a $54 \%$ (FAO, 2004). NRC (2001) reporta que el heno de avena cosechado a inicio de floración tiene $9.1 \%$ de PC, $58.0 \%$ de FDN y $1.10 \mathrm{Mcal} \mathrm{kg}^{-1} \mathrm{de} \mathrm{EN}_{\mathrm{L}}$. Sin embargo, cuando la avena se corta en etapa de madurez fisiológica se puede obtener un forraje de buena calidad, por efecto de dilución de la fibra al aumentar la proporción de grano de la planta (Cherney y Marten, 1982; Khorasani et al., 1997; Rosser et al., 2013). No obstante, se ha propuesto cosechar la avena entre las etapas de grano lechoso y masoso para optimizar el rendimiento y la calidad del forraje (Dumont et al., 2005; Espitia et al., 2012).

En el Estado de Chihuahua el cultivo de avena se establece en suelo plano (sin surcos), ya sea al voleo o con sembradora de cereales. En terrenos con desnivel se pierde agua por escurrimiento y humedad del suelo, y cuando se presentan lluvias torrenciales hay erosión, asolvamiento $y$ arrastre de nutrientes y semillas, lo que se traduce en bajos rendimientos (Ortiz, 2004). Ávila et al. (2006) indican que a estos factores deben agregarse la escasa y mala distribución de la precipitación, y la incapacidad de los suelos para retener la humedad. Una alternativa para incrementar la captación de agua en el suelo es implementar surcos con contras después de la siembra, porque permite conservar el suelo y reducir el escurrimiento de agua, especialmente en zonas áridas y semiáridas donde se presentan lluvias torrenciales y de poca duración (Ortiz, 2004). En otras latitudes, esta tecnología, comparada con el método convencional, ha mostrado incrementos en la producción de sorgo (Sorghum bicolor L. Moench) de $264 \%$ (1700 kg ha-1), $88 \%(1230 \mathrm{~kg}$ ha $^{-1}$ ) (Jones y Clark, 1987) y $94 \%\left(1390 \mathrm{~kg} \mathrm{ha}^{-1}\right)$ (Brhane et al., 2006), y en maíz (Zea mays L.) de $15.2 \%$ (1400 kg ha-1) (Howell et al., 2002).

De 1961 a la fecha el Instituto Nacional de Investigaciones Forestales, Agrícolas y Pecuarias (INIFAP) ha liberado 21 variedades de avena y publicado información respecto a: su origen genético y características vegetativas; su adaptación a diferentes ambientes; el desarrollo de variedades de ciclo corto $(90 \mathrm{~d})$ con buen rendimiento de forraje y gra- no, y con tolerancia a la roya del tallo (Puccinia graminis $\mathrm{F}$. sp.) (Ávila et al., 2006; Espitia et al., 2007). Pero a pesar de que la región noroeste del Estado de Chihuahua es prioritaria para producir el forraje de avena que se usa para alimentar 450000 cabezas de ganado, la investigación sobre rendimiento, composición química y valor nutricional del forraje de antiguas y nuevas variedades de avena es escasa. Tampoco existe información sobre los efectos de la siembra de las nuevas variedades en surcos con contras, ni sobre la mejor etapa de madurez al corte para lograr el mejor rendimiento y composición química del forraje.

El objetivo de este estudio fue evaluar el efecto del sistema de siembra en surcos con contras (también llamado "pileteo") y de la etapa de madurez al corte, sobre el rendimiento y composición química del heno de nuevas variedades de avena, bajo condiciones de temporal en el noroeste del Estado de Chihuahua.

\section{MATERIALES Y MÉTODOS}

El estudio se efectuó en cinco localidades del noroeste del Estado de Chihuahua, localizadas en la región conocida como la Baja Babícora, principal zona productora de avena en México. Esta región se ubica entre las coordenadas geográficas $28^{\circ} 15^{\prime}$ a $29^{\circ} 16^{\prime} \mathrm{LN}$ y $106^{\circ} 50^{\prime}$ a $107^{\circ} 29^{\prime} \mathrm{LO}$, con altitudes entre 1423 y $2210 \mathrm{~m}$. La precipitación promedio anual es de $438.8 \mathrm{~mm}$, con isoyetas de 280 a $600 \mathrm{~mm}$ durante la estación de crecimiento que inicia la tercera semana de junio (Ramírez y Ávila, 1996). La temperatura media anual de la zona es de $13.7^{\circ} \mathrm{C}$, con mínima de $6.2^{\circ} \mathrm{C}$ y máxima de $20.8^{\circ} \mathrm{C}$; y enero como el mes más frío.

Las localidades fueron Lázaro Cárdenas (Municipio de Cuauhtémoc), Santo Tomás (Guerrero), Rancho Santa Ana (Guerrero), Chopeque (Cusihuiriachi) y Rancho Teseachi (Namiquipa). Las fechas de siembra variaron del 24 de julio al 2 de agosto de 2005, con 'Cuauhtémoc' y 'Babícora' como variedades testigo más cinco nuevas variedades 'Karma', 'Cevamex', 'Menonita,' 'Teporaca' y 'Bachíniva'. Las variedades fueron seleccionadas por su adaptación y buen comportamiento a ambientes de Chihuahua y por ser de ciclo precoz a intermedio, adecuadas para la región avenera del estado que dispone de $92 \mathrm{~d}$ libres de heladas. Por las variaciones climáticas y de suelo (Cuadro 1), en cada localidad se establecieron dos parcelas de 0.25 ha; en una la siembra se hizo en suelo plano sin surcos (PSS), y en la otra en surcos con contras (SCC). Las parcelas se dividieron en tres sub-parcelas de $833 \mathrm{~m}^{2}$ a las que se les asignó al azar tres fechas de corte [en las etapas de embuche (EMB; $47 \pm$ $2 \mathrm{~d}$ ), grano masoso (MAS; $78 \pm 4 \mathrm{~d}$ ) y madurez fisiológica del grano (MF; $92 \pm 3 \mathrm{~d}$ ). En cada subparcela se delimitaron sub-sub-parcelas de $119 \mathrm{~m}^{2}$ donde las siete variedades se distribuyeron al azar. El diseño experimental fue uno de 
parcelas sub-sub-divididas en bloques completos al azar, en el que la localidad correspondió a la repetición, el sistema de siembra a la parcela grande, el estado de madurez al corte a la parcela mediana, y la variedad a la parcela chica. La densidad de siembra fue de $100 \mathrm{~kg} \mathrm{ha}^{-1}$ de semilla y la dosis de fertilización de 30N-40P-00K (en kg ha-1).

Para determinar el rendimiento de materia seca (MS) en $\mathrm{kg} \mathrm{ha}^{-1}\left(\mathrm{MS} \mathrm{ha}^{-1}\right)$, en cada parcela se lanzó al azar y en cinco ocasiones un marco cuadrado de madera de $1.0 \times 1.0 \mathrm{~m}$. El total de plantas (planta entera; tallos, hojas y granos) ubicadas dentro del cuadro se cortaron y pesaron; posteriormente se mezclaron y una submuestra de $1000 \mathrm{~g}$ se dejó en la parcela por $10 \mathrm{~d}$ para simular su henificación. El secado se completó en una estufa de aire forzado a $60^{\circ} \mathrm{C}$ por $24 \mathrm{~h}$. Posteriormente, las muestras fueron molidas en un molino Wiley ${ }^{\circledR}$ con malla de $1 \mathrm{~mm}$ (Arthur H. Tomas, Philadelphia, PA, USA).

A las muestras molidas se les determinó el contenido de MS total a $105^{\circ} \mathrm{C}$ por $12 \mathrm{~h}$, para expresar el contenido de nutrientes en base seca (\% de MS). Después las muestras se incineraron durante $2 \mathrm{~h}$ a $600{ }^{\circ} \mathrm{C}$ para obtener el contenido de materia orgánica y cenizas. La concentración de proteína cruda (PC) se midió por el método Kjeldahl ( $\mathrm{N}$ x 6.25) (AOAC, 1990). Las concentraciones de fibra detergente neutro (FDN), fibra detergente ácido (FDA) (Van Soest et al., 1991) y de lignina detergente ácido (LDA) (Goering y Van Soest, 1970), se obtuvieron secuencialmente en al analizador de fibras $\mathrm{ANKOM}^{200}{ }^{\circledR}$ (Ankom Technology, Fairport, NY, USA), mediante el uso de bolsas filtro Ankom ${ }^{\circledR}$ F57 con un tamaño de poro de 30 micrones. Para determinar la FDN se utilizó sulfito de sodio $\left(\mathrm{Na}_{2} \mathrm{SO}_{3}\right)$ y a-amilasa para remover el $\mathrm{N}$ y el almidón de la muestra, respectivamente. La hemicelulosa (HC) y celulosa (CL) se calcularon mediante la diferencia entre FDN y FDA, y entre FDA y lignina, respectivamente. La materia seca digestible (MSD) se estimó por la ecuación: $M S D(\%)=88.9-[0.779 \times(F D A, \%$ $M S)]$ (Moore y Undersander, 2002), y la energía neta de lac- tancia $\left(\mathrm{EN}_{\mathrm{L}}\right)$ mediante: $E N_{L}\left(\mathrm{Mcal}_{\mathrm{lb}} \mathrm{l}^{-1}\right)=0.7936-[0.00344$ $x$ (FDA, \% MS)] (Undersander et al., 1993) ajustada a Mcal $\mathrm{kg}^{-1}$ de MS.

Para explicar el efecto que tiene la relación grano y follaje sobre la calidad del heno de planta entera, del sistema PSS en la localidad Teseachi se seleccionaron 10 plantas al azar en cada etapa de corte de las variedades 'Bachíniva' y 'Teporaca'. En éstas se separó manualmente el grano (junto con espiguillas) y el follaje (hojas, tallos y raquis), y con los procedimientos antes descritos en cada fracción se determinaron los contenidos de PC, FDN, FDA y LDA. Este análisis sólo se hizo con dos tratamientos, debido al costo que representaba hacerlo con todos.

Los datos se analizaron con el procedimiento MIXED del SAS 9.1 (SAS, 2004) con base en un modelo mixto con efectos fijos (sistema de siembra, etapa de corte, sistema de siembra $\mathrm{x}$ etapa de corte, variedad, sistema de siembra $\mathrm{x}$ variedad, etapa de corte $\mathrm{x}$ variedad $\mathrm{y}$ sistema de siembra $\mathrm{x}$ etapa de corte $\mathrm{x}$ variedad) y efectos aleatorios (localidad o bloque, interacción sistema de siembra $\mathrm{x}$ localidad y sistema de siembra $\mathrm{x}$ etapa de corte $\mathrm{x}$ localidad). Cada variable se analizó por separado, de manera univariada. La comparación de medias se efectuó con la diferencia mínima significativa (DMS, $\alpha \leq 0.05$ ).

\section{RESULTADOS Y DISCUSIÓN}

Los análisis de varianza (Cuadro 2) indicaron que en todas las variables medidas el factor sistema de siembra no tuvo efecto significativo ni tuvo interacción con los factores variedad y etapa de madurez $(\mathrm{P}>0.05)$. La etapa de madurez y la variedad mostraron efectos significativos ( $\mathrm{P}$ $<0.01)$ en todas las variables, con excepción del contenido de lignina que no mostró diferencia entre niveles de madurez al corte. Se observó efecto de interacción $(\mathrm{P}<0.01)$ entre madurez y variedad para las variables proteína cruda (PC), fibra (FDN) y hemicelulosa (HC). Dada la ausencia

Cuadro 1. Descripción de variables del suelo y precipitación pluvial total ocurrida en las localidades durante la estación de crecimiento de las variedades de avena.

\begin{tabular}{lccccccc}
\hline Localidad & $\begin{array}{c}\text { Nitratos } \\
\left(\mathrm{kg} \mathrm{ha}^{-1}\right)\end{array}$ & Fósforo (ppm) & $\begin{array}{c}\text { Materia } \\
\text { orgánica (\%) }\end{array}$ & Saturación (\%) & $\begin{array}{c}\mathrm{pH} \mathrm{H}_{2} \mathrm{O} \\
(1: 20)\end{array}$ & Textura & $\begin{array}{c}\text { Precipitación } \\
\text { pluvial }(\mathrm{mm})\end{array}$ \\
\hline Lázaro Cárdenas & 15.2 & 21.34 & 1.745 & 35.0 & 5.26 & Franco & 149 \\
Santo Tomás & 9.5 & 29.88 & 0.838 & 27.5 & 5.36 & $\begin{array}{c}\text { Franco arenoso } \\
277\end{array}$ \\
Santa Ana & 10.8 & 50.12 & 1.466 & 34.5 & 5.11 & $\begin{array}{c}\text { Franco arcillo } \\
\text { arenoso }\end{array}$ & 260 \\
Chopeque & 38.9 & 36.22 & 1.815 & 30.0 & 5.11 & Franco arenoso & 180 \\
Teseachi & 37.6 & 19.28 & 1.815 & 61.5 & 7.92 & Franco & 503 \\
\hline
\end{tabular}

Profundidad de muestreo del suelo, 0 a $20 \mathrm{~cm}$. 
de efectos del sistema de siembra, en los cuadros se omiten las medias de este factor.

\section{Rendimiento de forraje}

A diferencia de lo reportado en otros estudios (Jones y Clark, 1987; Brhane et al., 2006), en esta investigación no se observó $(\mathrm{P}=0.9662)$ relación entre el sistema de siembra y las variedades en cuanto al rendimiento de forraje. La falta de efecto del sistema de siembra surco con contras (SCC) sobre el rendimiento de forraje puede explicarse por la destrucción parcial de las contras o piletas causada por lluvias torrenciales ocurridas cuando aún no estaban bien consolidadas; además, como la mayor precipitación durante el experimento ocurrió en las etapas tempranas del crecimiento vegetativo (519 $\mathrm{mm}$ o $38 \%$ al amacollo, $421 \mathrm{~mm}$ o $31 \%$ al embuche, $146 \mathrm{~mm}$ o $11 \%$ en floración, y $284 \mathrm{~mm}$ o $21 \%$ en llenado del grano), era de esperarse un mejor desarrollo de la planta y mayor producción de biomasa que en condiciones de menor precipitación, como propusieron Jones y Clark (1987). La escasa pendiente de los terrenos pudo contribuir también a la falta de respuesta. En sorgo, Gerard et al. (1984) evaluaron el efecto del contreo en surco, y observaron que la producción fue 302, 140 y 42 \% más que el testigo cuando la pendiente del suelo fue alta, media y baja, respectivamente.

Cuando el corte se hizo en la etapa de madurez (MF) el rendimiento de materia seca fue $259 \%$ más que en embuche (EMB) y 43 \% más que en grano masoso (MAS); mientras que MAS superó en $150 \%$ a EMB (Cuadro 3). En otros estudios también se observó que el mayor rendimiento se alcanza entre las etapas de floración y de grano lechoso, para después disminuir en las etapas de lechoso a masoso
(FAO, 2004). Johnston et al. (1999) reportaron que los rendimientos de biomasa de avena en MAS fueron más altos que en $\mathrm{EMB}$, con incrementos de hasta $110 \%$; este resultado coincide con lo observado en el presente estudio en el que el respectivo aumento fue de $150 \%$. Esto confirma que la mayor producción de biomasa se obtiene en etapas anteriores a la madurez fisiológica, ya que el registrado en MAS representa hasta $70 \%$ del total de forraje producido, tal como ocurrió en el presente estudio (Cuadro 3).

El rendimiento de $4475 \mathrm{~kg}$ de $\mathrm{MS} \mathrm{ha}^{-1}$ aquí obtenido cuando la cosecha se efectuó en la etapa de MF, es cercano al de $5000 \mathrm{~kg}$ de $\mathrm{MS} \mathrm{ha}^{-1}$ reportado por Salmerón et al. (2003) en la misma etapa de corte. Al respecto, la media histórica de producción de forraje de avena de temporal en México es de $2800 \mathrm{~kg}$ de MS ha-1.

'Karma' fue la única variedad que mostró menor rendimiento $(\mathrm{P}<0.05)$ (Cuadro 3), lo cual se debió probablemente a la baja viabilidad de su semilla y la consecuente disminución en densidad de población, ya que en otros estudios 'Karma' ha mostrado rendimientos similares a las demás variedades aquí evaluadas (Salmerón et al., 2003). La ausencia de diferencias en el rendimiento entre las otras variedades, que incluyen a las nuevas y los testigos, pudo deberse a que la precipitación promedio de las localidades fue $343 \mathrm{~mm}$, superior a la condición de precipitación intermedia (200 a $300 \mathrm{~mm}$ ) considerada como suficiente para el óptimo desarrollo del cultivo de avena en la región (Salmerón, 2000; Salmerón et al., 2003); en este intervalo de lluvias se han reportado rendimientos hasta de $6200 \mathrm{~kg}$ de MS ha-1 (Salmerón, 2000).

Cuadro 2. Resumen del análisis de varianza del efecto del sistema de siembra (sistema), etapa de madurez al corte (madurez) y variedad sobre el rendimiento de materia seca y composición química del heno de siete variedades de avena.

\begin{tabular}{|c|c|c|c|c|c|c|c|c|c|c|}
\hline Factor & GL & Rendimiento & PC & FDN & FDA & LDA & $\mathrm{HC}$ & $\mathrm{CL}$ & MSD & $\mathrm{EN}_{\mathrm{L}}$ \\
\hline Sistema & 1 & ns & ns & ns & ns & ns & ns & ns & ns & ns \\
\hline Madurez & 2 & ** & ** & ** & ** & ns & ** & ** & ** & ** \\
\hline Sistema $\mathrm{x}$ madurez & 2 & ns & ns & ns & ns & ns & ns & ns & ns & ns \\
\hline Variedad & 6 & ** & ** & ** & ** & ** & $* *$ & $* *$ & ** & $* *$ \\
\hline Sistema $\mathrm{x}$ variedad & 6 & ns & ns & ns & ns & ns & ns & ns & ns & ns \\
\hline Madurez x variedad & 12 & ns & $* *$ & $* *$ & ns & ns & $* *$ & ns & ns & ns \\
\hline Sistema $\mathrm{x}$ madure $\mathrm{x}$ variedad & 12 & ns & ns & ns & ns & ns & ns & ns & ns & ns \\
\hline Lineal $^{\dagger}$ & 16 & ** & $* *$ & ** & $* *$ & ns & $* *$ & $* *$ & $* *$ & $* *$ \\
\hline Cuadrático $^{\dagger}$ & 16 & ns & $* *$ & ns & ns & ns & ns & ns & ns & ns \\
\hline
\end{tabular}

$\mathrm{GL}=$ grados de libertad; ${ }^{\star}=\mathrm{P} \leq 0.05 ;{ }^{\star \star}=\mathrm{P} \leq 0.01 ; \mathrm{ns}=$ no significativo; ${ }^{\dagger}$ Contraste polinomial, lineal o cuadrático, para el efecto de madurez al corte; $\mathrm{PC}=$ proteína cruda; FDN = fibra detergente neutro; FDA = fibra detergente ácido; LDA = lignina; $\mathrm{HC}=$ hemicelulosa; $\mathrm{CL}=$ celulosa; $\mathrm{MSD}=$ materia seca digestible; $\mathrm{EN}_{\mathrm{L}}=$ energía neta de lactancia. 
Cuadro 3. Efecto de la etapa de madurez al corte y variedad sobre el rendimiento de materia seca $\left(\mathrm{kg} \mathrm{ha}^{-1}\right)$ y contenido de proteína cruda (PC, \% de la MS) en el heno de siete variedades de avena.

\begin{tabular}{|c|c|c|c|c|c|c|c|}
\hline \multirow{2}{*}{ Variedad } & \multicolumn{4}{|c|}{ MS $\left(\mathrm{kg} \mathrm{ha}^{-1}\right)$} & \multicolumn{3}{|c|}{ PC (\%) } \\
\hline & EMB & MAS & MF & Media & EMB & MAS & MF \\
\hline 'Bachíniva' & 1144 & 3220 & 4448 & 2937 a & $17.6 \mathrm{ab}$ & $11.3 \mathrm{ab}$ & $10.9 \mathrm{ab}$ \\
\hline 'Menonita' & 1325 & 3353 & 4490 & $3056 \mathrm{a}$ & $17.4 \mathrm{ab}$ & $10.6 \mathrm{abc}$ & $10.5 \mathrm{ab}$ \\
\hline 'Teporaca' & 1243 & 3128 & 4740 & $3037 \mathrm{a}$ & $18.2 \mathrm{a}$ & $11.6 \mathrm{ab}$ & $10.3 \mathrm{ab}$ \\
\hline 'Karma’ & 957 & 2674 & 4148 & $2593 \mathrm{~b}$ & $18.5 \mathrm{a}$ & $11.4 \mathrm{ab}$ & $10.4 \mathrm{ab}$ \\
\hline ‘Cevamex’ & 1225 & 3013 & 4753 & $2998 \mathrm{a}$ & $18.4 \mathrm{a}$ & $11.8 \mathrm{a}$ & $9.9 \mathrm{~b}$ \\
\hline 'Cuauhtémoc' & 1565 & 3148 & 4426 & $3047 a$ & $15.4 \mathrm{c}$ & $10.5 \mathrm{bc}$ & $11.3 \mathrm{a}$ \\
\hline 'Babícora' & 1269 & 3302 & 4320 & $2964 \mathrm{a}$ & $16.6 \mathrm{bc}$ & $9.7 \mathrm{c}$ & $10.9 \mathrm{ab}$ \\
\hline EE de la media & 354 & 354 & 354 & 311 & 1.0 & 1.0 & 1.0 \\
\hline Media $^{\dagger}$ & $1247 \pm 323 \mathrm{C}$ & $3120 \pm 323 \mathrm{~B}$ & $4475 \pm 323 \mathrm{~A}$ & & $17.4 \pm 0.93 \mathrm{~A}$ & $11.0 \pm 0.93 \mathrm{BC}$ & $10.6 \pm 0.93 \mathrm{C}$ \\
\hline
\end{tabular}

Para cada variable, medias con letras minúsculas iguales dentro de columnas, o mayúsculas en hileras, no son estadísticamente diferentes (DMS, 0.05). Corte de avena en las etapas de embuche (EMB), grano masoso (MAS), madurez fisiológica del grano (MF); ${ }^{\dagger}$ Media \pm error estándar.

Por su parte, Ávila et al. (2006) observaron que en ocho genotipos de avena cultivados en condiciones de temporal malo, regular y bueno, el coeficiente de variación fue 39, 12 y $8 \%$, respectivamente. Es probable que las diferencias en rendimiento entre variedades sean mayores en condición de temporal con poca lluvia (menos de $200 \mathrm{~mm}$ ) que en condiciones de temporal intermedio o favorable (más de $300 \mathrm{~mm}$ de lluvia).

\section{Composición química}

\section{Proteína cruda (PC)}

La PC promedio de las siete variedades disminuyó en seis unidades porcentuales (equivalente a $37 \%$ ) al pasar de la etapa de EMB a la de MAS, y luego se incrementó media unidad porcentual (equivalente a $3 \%$ ) al pasar de MAS a la etapa de MF $(\mathrm{P}<0.05)$ (Cuadro 3). Este es un comportamiento normal pues si bien en las etapas vegetativas es rápida la absorción de nitrógeno por el sistema radical de las gramíneas, cuando la planta acelera su crecimiento el $\mathrm{N}$ se diluye debido a que el incremento de MS no-nitrogenada supera a la absorción de este elemento. Al respecto, Johnston et al. (1999) encontraron que el descenso de PC en forraje de avena en etapas de corte similares a las del presente estudio (EMB a MAS) estuvo entre 40 y $50 \%$; otros autores han reportado reducciones de $54 \%$ (166 a $76 \mathrm{~g} \mathrm{~kg}^{-1}$ de MS) (Dumont et al., 2005) y $56 \%$ (173 a $76 \mathrm{~g} \mathrm{~kg}^{-1}$ de MS) (Espitia et al., 2012).

La interacción variedad $\mathrm{x}$ etapa de madurez al corte fue significativa para PC (Cuadro 2), y en promedio hubo una disminución de tipo cuadrática al avanzar a estados de mayor madurez, con coeficientes distintos entre algunas va- riedades $(\mathrm{P}<0.05)$ (Cuadro 3). La interacción implica que las variedades 'Bachíniva, 'Menonita, 'Teporaca' y 'Karma' no difieren entre sí en el contenido de PC, de manera que pueden cosecharse en cualquier etapa de madurez; en cambio, en la variedad 'Cevamex' el contenido de PC es menor que en otras variedades cuando se cosecha en la etapa de MF, mientras que en las variedades 'Cuauhtémoc' y 'Babícora' el contenido de $\mathrm{PC}$ es mayor cuando se cosechan en MF (Cuadro 3). Lo anterior sugiere que, excepto 'Cevamex', cualquier variedad puede sembrarse si se va a cosechar en la etapa de madurez fisiológica del grano, que es como tradicionalmente se hace en esta entidad.

Al comparar los resultados del presente estudio (Cuadro 3) con los de Espitia et al. (2012), las variedades 'Cevamex', 'Karma' y 'Chihuahua' (ésta última muy similar a la variedad 'Cuauhtémoc') presentaron 1.3, 0.9 y 2.4 unidades menos de PC en la etapa de EMB, pero 2.5, 2.8 y 3.4 unidades más en MF. Si la comparación se hace con los resultados de Salmerón et al. (2003), en la etapa de MF las siete variedades presentaron en promedio $10.6 \%$ de PC, similar al valor de $10.7 \%$ observado por estos autores con las mismas variedades, pero superior al promedio de $9.6 \%$ de variedades antiguas como 'Guelatao', 'Tarahumara, 'Páramo' y 'Diamante'. La variedad 'Cuauhtémoc' presentó $11.3 \%$ de PC, similar al $11.5 \%$ reportado por Salmerón et al. (2003). Esta variedad forrajera ha sido desde hace mucho tiempo, una buena opción para los productores de la región; no obstante, su alta susceptibilidad a las razas de roya ha obligado a remplazarla por variedades resistentes como 'Teporaca', 'Menonita' y 'Bachiniva'.

Con base en los porcentajes promedio de grano y follaje en la planta de avena y su respectiva composición química 
(Cuadro 4), se deduce que en las últimas dos etapas de madurez el contenido de PC depende mayormente de la cantidad de grano presente en la planta, mientras que el del follaje (hojas y tallos) presenta niveles de 4 a $5 \%$, lo cual es típico en gramíneas durante la etapa de madurez avanzada. Es importante señalar que esta información corresponde al promedio de dos variedades ('Bachíniva' y 'Teporaca') sembradas en una sola localidad (Teseachi), donde ocurrió la mayor precipitación.

\section{Fibra detergente neutro (FDN) y fibra detergente ácido (FDA)}

La FDN resultó afectada por la interacción variedad $\mathrm{x}$ etapa de madurez al corte $(\mathrm{P}<0.05$, Cuadro 2$)$. En EMB las variedades 'Teporaca' y 'Cevamex' fueron las de menor contenido de FDN, mientras que en MAS y MF fueron 'Teporaca' y 'Karma'. Por su parte, la FDA mostró efecto de variedad $(\mathrm{P}<0.05)$ y etapa de corte $(\mathrm{P}<0.05)$, y 'Karma' fue la variedad con menor contenido de FDA (Cuadro 5).

El contenido de FDN y FDA cambia al avanzar la madurez de las plantas de avena; valores de 53.7 a $62.4 \%$ y 35.2 a $43.3 \%$ (Johnston et al., 1999) y de 50.8 a $62.2 \%$ y 24.9 a $34.2 \%$ (Coblentz et al., 2000) han sido reportados en plantas cortadas en EMB y MAS, respectivamente. Los mismos autores afirmaron que FDN y FDA aumentan entre $15 \mathrm{y}$ $25 \%$ de EMB a MAS, pero que la calidad y digestibilidad del heno de avena declina al entrar a la etapa de floración. Contrariamente a lo citado por estos autores, en este estu- dio la FDN y FDA disminuyeron linealmente $(\mathrm{P}<0.05)$ en 5.0 y 3.4 unidades porcentuales, respectivamente, al pasar de una etapa de madurez a otra (Cuadro 5). Al respecto, es sabido que en avena el contenido de proteína cruda se reduce al avanzar la madurez de la planta y el contenido de fibra se incrementa hasta iniciada la formación del grano; posteriormente la fibra tiende a decrecer en las siguientes etapas fenológicas como resultado del llenado del grano y la consecuente acumulación de almidón, pero mantiene su calidad (Khorasani et al., 1997; Rosser et al., 2013). Esto se explica por el aumento en la proporción de grano y su bajo contenido de fibra (Cuadro 4), que superan al aumento del contenido de FDN y FDA en el follaje (Cherney y Marten, 1982), por lo que en la planta completa la fibra se diluye al pasar de EMB, a MAS y MF (Cuadro 4).

\section{Hemicelulosa (HC) y celulosa (CL)}

Las fracciones de HC (Cuadro 6) y CL mostraron un comportamiento similar al de las fibras FDN y FDA (Cuadro 2), respectivamente. El contenido de $\mathrm{HC}$ mostró efecto de la interacción variedad $\mathrm{x}$ etapa de madurez (Cuadros 2 y 6), con un descenso lineal al avanzar la madurez $(\mathrm{P}<$ 0.05), con excepción de 'Cevamex'. Las variedades con menor contenido de CL fueron 'Karma', 'Teporaca' y 'Bachíniva' (datos no mostrados). En promedio ambas fracciones disminuyeron $(\mathrm{P}<0.05) 1.5$ y 3.35 unidades porcentuales de una etapa de madurez a otra, con medias para HC de $24.8,24.1$ y $22.1 \%$, y para CL de $30.5,26.4$ y $23.8 \%$ en EMB, MAS y MF, respectivamente.

Cuadro 4. Porcentaje de grano y follaje y su relación con la composición química (\% de la MS) en el heno de avena cosechado en tres etapas de madurez ${ }^{\dagger}$.

\begin{tabular}{|c|c|c|c|c|c|c|}
\hline \multirow{2}{*}{ Variable } & \multicolumn{2}{|c|}{ EMB } & \multicolumn{2}{|c|}{ MAS } & \multicolumn{2}{|c|}{ MF } \\
\hline & Grano & Follaje & Grano & Follaje & Grano & Follaje \\
\hline Porcentaje de grano y follaje $\mathrm{e}^{\dagger}$ & 17.2 & 82.8 & 51.7 & 48.3 & 56.1 & 43.9 \\
\hline PC & 25.4 & 18.7 & 15.1 & 9.2 & 15.8 & 5.4 \\
\hline FDN & 53.0 & 55.3 & 23.5 & 62.0 & 19.1 & 67.5 \\
\hline FDA & 23.6 & 33.4 & 10.5 & 37.4 & 8.2 & 40.3 \\
\hline \multirow[t]{2}{*}{ LDA } & 2.30 & 2.60 & 1.40 & 3.70 & 1.00 & 4.20 \\
\hline & \multicolumn{6}{|c|}{ Planta completa } \\
\hline PC & \multicolumn{2}{|c|}{19.8} & \multicolumn{2}{|c|}{12.3} & \multicolumn{2}{|c|}{11.2} \\
\hline FDN & \multicolumn{2}{|c|}{55.0} & \multicolumn{2}{|c|}{42.1} & \multicolumn{2}{|c|}{40.4} \\
\hline FDA & \multicolumn{2}{|c|}{31.7} & \multicolumn{2}{|c|}{23.5} & \multicolumn{2}{|c|}{22.3} \\
\hline LDA & \multicolumn{2}{|c|}{2.5} & \multicolumn{2}{|c|}{2.5} & \multicolumn{2}{|c|}{2.4} \\
\hline
\end{tabular}

Corte de avena en las etapas de embuche (EMB), grano masoso (MAS), madurez fisiológica del grano $(\mathrm{MF}) . \mathrm{PC}=$ proteína cruda, FDN $=$ fibra detergente neutro, FDA = fibra detergente ácido, $\mathrm{LDA}=$ lignina detergente ácido; Grano = granos + espiguillas; Follaje = hojas + tallos + raquis. ${ }^{\dagger}$ Promedio de las variedades 'Bachíniva' y 'Teporaca', del sistema plano sin surco en la localidad de Teseachi. 
Cuadro 5. Efecto de la etapa de corte y variedad sobre el contenido (\% de la MS) de fibra detergente neutro (FDN) y fibra detergente ácido (FDA) en el heno de siete variedades de avena.

\begin{tabular}{|c|c|c|c|c|c|c|c|}
\hline \multirow{2}{*}{ Variedad } & \multicolumn{3}{|c|}{ FDN (\%) } & \multicolumn{3}{|c|}{ FDA (\%) } & \multirow{2}{*}{ Media } \\
\hline & EMB & MAS & MF & EMB & MAS & MF & \\
\hline 'Bachíniva' & $59.0 \mathrm{a}$ & $51.5 \mathrm{bc}$ & $44.9 \mathrm{~d}$ & 33.4 & 28.6 & 24.8 & $28.9 \mathrm{cde}$ \\
\hline 'Menonita' & $58.4 \mathrm{ab}$ & $54.1 \mathrm{a}$ & $50.2 \mathrm{a}$ & 33.8 & 29.9 & 27.4 & $30.4 \mathrm{a}$ \\
\hline 'Teporaca' & $55.3 \mathrm{c}$ & $51.0 \mathrm{bc}$ & $46.3 \mathrm{~cd}$ & 31.7 & 28.1 & 25.2 & $28.3 \mathrm{de}$ \\
\hline 'Karma' & $56.6 \mathrm{abc}$ & $50.3 c$ & $45.2 \mathrm{~d}$ & 32.7 & 27.4 & 24.4 & $28.2 \mathrm{e}$ \\
\hline ‘Cevamex’ & $55.1 \mathrm{c}$ & $53.1 \mathrm{ab}$ & $48.6 \mathrm{ab}$ & 31.8 & 29.2 & 26.6 & $29.2 \mathrm{bcd}$ \\
\hline 'Cuauhtémoc' & $56.5 \mathrm{bc}$ & $52.5 \mathrm{abc}$ & $47.8 \mathrm{bc}$ & 32.9 & 29.3 & 26.3 & $29.5 \mathrm{bc}$ \\
\hline ‘Babícora’ & $58.7 \mathrm{ab}$ & $51.7 \mathrm{bc}$ & $48.6 \mathrm{ab}$ & 33.7 & 29.2 & 27.2 & $30.0 \mathrm{ab}$ \\
\hline EE de la media & 1.49 & 1.49 & 1.49 & 0.87 & 0.87 & 0.87 & 0.60 \\
\hline $\mathrm{Media}^{\dagger}$ & $57.1 \pm 1.27 \mathrm{~A}$ & $52.0 \pm 1.27 \mathrm{~B}$ & $47.4 \pm 1.27 \mathrm{C}$ & $32.8 \pm 0.7 \mathrm{~A}$ & $28.8 \pm 0.7 \mathrm{~B}$ & $26.0 \pm 0.7 \mathrm{C}$ & \\
\hline
\end{tabular}

Promedios con letras minúsculas iguales dentro de columnas o mayúsculas en hileras no son estadísticamente diferentes (DMS, 0.05). Corte de avena en las etapas de embuche (EMB), grano masoso (MAS), madurez fisiológica del grano (MF). ${ }^{\dagger}$ Media \pm error estándar.

Cuadro 6. Efecto de la etapa de madurez al corte y variedad sobre el contenido (\% de la MS) de hemicelulosa (HC) y lignina (LDA) en el heno de siete variedades de avena.

\begin{tabular}{|c|c|c|c|c|c|c|c|}
\hline \multirow{2}{*}{ Variedad } & \multicolumn{3}{|c|}{$\mathrm{HC}(\%)$} & \multicolumn{3}{|c|}{ LDA (\%) } & \multirow{2}{*}{ Media } \\
\hline & EMB & MAS & MF & EMB & MAS & MF & \\
\hline 'Bachíniva' & $26.1 \mathrm{a}$ & $23.8 \mathrm{c}$ & $20.9 \mathrm{~d}$ & 2.6 & 2.7 & 2.5 & $2.61 \mathrm{bc}$ \\
\hline 'Menonita' & $25.4 \mathrm{abc}$ & $25.1 \mathrm{a}$ & $23.4 \mathrm{a}$ & 2.6 & 3.0 & 2.8 & $2.81 \mathrm{a}$ \\
\hline 'Teporaca' & $24.2 \mathrm{~d}$ & $24.1 \mathrm{bc}$ & $21.8 \mathrm{~cd}$ & 2.6 & 2.7 & 2.3 & $2.51 \mathrm{~cd}$ \\
\hline 'Karma’ & $24.5 \mathrm{~cd}$ & $23.7 \mathrm{c}$ & $21.5 \mathrm{~cd}$ & 2.5 & 2.6 & 2.3 & $2.47 \mathrm{~d}$ \\
\hline 'Cevamex’ & $23.9 \mathrm{~d}$ & $24.8 \mathrm{ab}$ & $22.8 \mathrm{ab}$ & 2.4 & 2.7 & 2.6 & $2.53 \mathrm{bcd}$ \\
\hline 'Cuauhtémoc’ & $24.2 \mathrm{~d}$ & $24.1 \mathrm{bc}$ & $22.2 \mathrm{bc}$ & 2.6 & 2.6 & 2.5 & $2.58 \mathrm{bcd}$ \\
\hline Babícora & $25.6 \mathrm{ab}$ & $23.5 c$ & $22.1 \mathrm{bc}$ & 2.6 & 2.7 & 2.6 & $2.64 \mathrm{~b}$ \\
\hline EE de la media & 0.83 & 0.83 & 0.83 & 0.10 & 0.10 & 0.10 & 0.07 \\
\hline Media $^{\dagger}$ & $24.8 \pm 0.76 \mathrm{~A}$ & $24.1 \pm 0.76 \mathrm{AB}$ & $22.1 \pm 0.76 \mathrm{~B}$ & $2.56 \pm 0.08 \mathrm{~A}$ & $2.70 \pm 0.08 \mathrm{~A}$ & $2.51 \pm 0.08 \mathrm{~A}$ & \\
\hline
\end{tabular}

Promedios con letras minúsculas iguales dentro de columnas o mayúsculas en hileras no son estadísticamente diferentes (DMS, 0.05). Corte de avena en las etapas de embuche (EMB), grano masoso (MAS), madurez fisiológica del grano (MF). ${ }^{\dagger}$ Media \pm error estándar.

\section{Lignina detergente ácido (LDA)}

El contenido de LDA solamente mostró diferencias entre variedades $(\mathrm{P}<0.05)$, y las menores concentraciones ocurrieron en las variedades 'Karma' (2.47\%), 'Teporaca' (2.51 $\%)$, 'Cevamex' (2.53 \%) y 'Cuauhtémoc' (2.58 \%) (Cuadro 6). En cuanto al contenido de LDA en etapas fenológicas al corte, su concentración promedio en EMB $(2.56 \%)$ y MAS $(2.70 \%)$ fue mayor y menor a los valores de 0.65 y $5.0 \%$ reportados por Coblentz et al. (2000) en las mismas etapas fenológicas. Por su parte, el NRC (2001) reporta un valor de $6.5 \%$ para el heno de avena a inicio de floración. Las bajas concentraciones de LDA observadas en este estudio podrían suponer una alta digestibilidad de la fibra detergente neutro del forraje; no obstante, la mayor parte de esa
LDA se encontró en hojas y tallos (84.5\% en EMB, $71.7 \%$ en MAS y $76.2 \%$ en MF) (Cuadro 4). Los valores de LDA usualmente se correlacionan positivamente con la etapa de madurez al corte: $2.6 \%$ en EMB, $3.7 \%$ en MAS y $4.2 \%$ en MF, lo que afecta la disponibilidad de nutrientes en el contenido celular.

De manera similar, el contenido de fibra en la fracción follaje del heno de avena se incrementó linealmente a medida que la planta avanzaba en su desarrollo, para alcanzar su máximo en MF (Cuadro 4). Lo anterior es importante para determinar la época de corte de la avena, pues según Oben y Allen (1999), si el corte se hace en MF se maximizaría el rendimiento pero se cosecharía un heno cuya fracción follaje causaría disminuciones en consumo, digestibilidad 
y tasa de pasaje, en detrimento del desempeño productivo de los animales. Sin embargo, Wallsten et al. (2009) encontraron que las vaquillas alimentadas con ensilaje de avena cosechado en etapa de grano masoso, presentaron mayor consumo de materia seca y orgánica que las alimentadas con ensilaje cosechado en floración o grano lechoso. Estos últimos autores sugirieron que los animales tienden a seleccionar las partes menos fibrosas y más apetecibles del forraje (como el grano), lo que resulta en un mayor consumo de alimento.

En general, la composición química del forraje de avena está influenciada por varios factores, de los cuales la estación de crecimiento y la etapa de madurez al corte tienen mayor impacto (Coblentz et al., 2000; Coblentz y Walgenbach, 2010), especialmente cuando inicia la floración y el desarrollo del grano, que a su vez dependen de la variedad. En la variedad 'Vista' cortada en la etapa de EMB, Coblentz y Walgenbach (2010) reportaron contenidos de PC (17.0 \%), FDN (57.8\%), FDA (33.4\%), HC (24.4\%), CL (29.6\%) y LDA (2.68 \%), similares a los reportados en el presente estudio en la misma etapa. No obstante, es difícil encontrar similitudes en la composición química, excepto para PC, en subsiguientes etapas de madurez, aun dentro del mismo estado fenológico, debido a lo heterogéneo del forraje cuando se cosecha después de iniciada la floración y llenado del grano (Cherney y Marten, 1982).

\section{Materia seca digestible (MSD)}

En la variable MSD hubo efecto de la variedad $(\mathrm{P}<0.001)$ y de la etapa de madurez al corte $(\mathrm{P}<0.001)$ (Cuadro 2$)$. Las variedades con mayor $(\mathrm{P}<0.01)$ MSD fueron 'Karma', 'Teporaca' y 'Bachíniva' (datos no mostrados). La MSD se incrementó linealmente $\left(\mathrm{P}<0.05 ; \mathrm{R}^{2}=0.89\right)$ en 2.7 unidades porcentuales al avanzar la madurez, con promedios de $63.3,66.5$ y $68.7 \%$ para EMB, MAS y MF, respectivamente. Los valores de MSD de 63.3 y $66.5 \%$ observados en este estudio en EMB y MAS, difieren de los datos 71.9 y $55.0 \%$ obtenidos en Estados Unidos por Coblentz et al. (2000), o de los 75.2 y $57.2 \%$ reportados por FAO (2004). Esto puede explicarse por la mayor proporción de grano producido por las plantas de avena en MAS con respecto a EMB, pero con valores similares de lignina (Cuadro 4). En su estudio, Rosser et al. (2013) concluyeron que la cosecha de avena en etapa de madurez fisiológica del grano maximiza el rendimiento de materia seca efectiva degradable, en comparación con la cosecha en las etapas de floración, grano lechoso o grano masoso, como normalmente se hace en Estados Unidos y Canadá.

\section{Energía neta de lactancia $\left(\mathrm{EN}_{\mathrm{L}}\right)$}

La $\mathrm{EN}_{\mathrm{L}}$ mostró efecto de la etapa de madurez al corte $(\mathrm{P}$
$<0.01)$ y de variedad $(\mathrm{P}<0.01)$ (Cuadro 2). Las variedades con las concentraciones más altas $(\mathrm{P}<0.01)$ fueron 'Karma', 'Teporaca' y 'Bachíniva' (datos no mostrados). $\mathrm{La} \mathrm{EN}_{\mathrm{L}}$ aumentó $\left(\mathrm{P}<0.05 ; \mathrm{r}^{2}=0.989\right)$ en 0.03 unidades al avanzar la madurez, con promedios de $1.50 \pm 0.005,1.53 \pm 0.005$ y $1.55 \pm 0.005 \mathrm{Mcal} \mathrm{kg}^{-1}$ de MS para EMB, MAS y MF, respectivamente. Estos valores son superiores al valor de 1.10 Mcal kg-1 de MS reportado por NRC (2001) para heno de avena cosechado a inicio de floración. El aumento de valores de $\mathrm{EN}_{\mathrm{L}}$ conforme avanza la madurez se relacionó negativamente con el contenido de fibra, por el efecto de dilución debida a la relación positiva entre la proporción de grano y la madurez.

\section{CONCLUSIONES}

El sistema de siembra en surcos con contras no afectó el rendimiento ni la composición química del heno de avena, que en la etapa de madurez fisiológica del grano mostró el mayor rendimiento y la mejor calidad del forraje, atribuible a la mayor proporción de grano en la planta en esa etapa y a que diluyó los componentes fibrosos, no obstante que la concentración de fibra en la fracción follaje también alcanzó su valor máximo en la etapa de madurez fisiológica. Las variedades de avena no mostraron diferencias importantes en cuanto a su composición química.

\section{AGRADECIMIENTOS}

A la Fundación Produce, Chihuahua A. C., por el apoyo económico brindado para la realización de este estudio a través del proyecto número 08-2005-0982.

\section{BIBLIOGRAFÍA}

AOAC, Association of Official Analytical Chemists (1990) Official Methods of Analysis. 15th ed. Association of Official Analytical Chemists. Washington, DC, USA. 1298 p.

Ávila M M R, J J Salmerón Z (1999) Adopción de variedades de avena y su impacto en el Estado de Chihuahua. Folleto Científico No. 5. CESICH-CIRNOC-INIFAP-SAGAR. Ciudad Cuauhtémoc, Chihuahua, México. $38 \mathrm{p}$.

Ávila M M R, R Gutiérrez G, J J Salmerón Z, P Fernández H, D Domínguez D (2006) Diagnóstico del sistema de producción de avena temporal en Chihuahua. Folleto Técnico No. 22. CESICH-CIRNOC-INIFAP-SAGARPA. Ciudad Cuauhtémoc, Chihuahua, México. 43 p.

Brhane G, C S Wortmann, M Mamo, H Gebrekidan, A Belay (2006) Micro-basin tillage for grain sorghum production in semiarid areas of Northern Ethiopia. Agron. J. 98:124-128.

Cherney J H, G C Marten (1982) Small grain crop forage potential: II. Interrelationships among biological, chemical, morphological and anatomical determinants of quality. Crop Sci. 22:240-245.

Coblentz W K, K P Coffey, J E Turner, D A Scarbrough, J S Weyers, K F Harrison, Z B Johnson, L B Daniels, C F Rosenkrans Jr, D W Kellogg, D S Hubbell (2000) Effect of maturity on degradation kinetics of sod-seeded cereal grain forage grown in Northern Arkansas. J. Dairy Sci. 83:2499-2511.

Coblentz W K, R P Walgenbach (2010) Fall growth, nutritive value, and estimation of total digestible nutrients for cereal-grain forages in the north-central United States. J. Anim. Sci. 88:383-399. 
Dumont L J C, R G Anrique, C D Alomar (2005) Efecto de dos sistemas de determinación de materia seca en la composición química y calidad del ensilaje directo de avena en diferentes estados fenológicos. Agric. Téc. 65: 388-396.

Espitia R E, H E Villaseñor M, J H Espino, J J Salmerón Z, R M González I, L Osorio A (2007) Obsidiana, variedad de avena para la producción de grano y forraje en México. Agric. Téc. Méx. 33:95-98.

Espitia R E, H E Villaseñor M, R G Tovar, M de la O Olán, O A Limón (2012) Momento óptimo de corte para rendimiento y calidad de variedades de avena forrajera. Rev. Mex. Cien. Agríc. 3:771783.

FAO (2004) Food and Agriculture Organization of the United Nations. Fodder Oats; a World Overview. Agriculture Department. Plant Production and Protection, Series No. 33. J M Suttle, S G Reynolds (eds.). Disponible en: www.fao.org/docrep/008/ y5765e/y5765e00.htm. (Julio 2012).

Gerard C J, P D Sexton, D M Conover (1984) Effect of furrow diking, subsoiling, and slope position on crop yields. Agron. J. 76:945950.

Goering H K, P J Van Soest (1970) Forage Fiber Analysis (Apparatus, Reagents, Procedures, and some Applications). Agric. Handbook No. 379. USDA-ARS, Washinton, DC. 20 p.

Howell T A, A D Schneider, D A Dusek (2002) Effects of furrow diking on corn response to limited and full sprinkler irrigation. Soil Sci. Soc. Amer. J. 66:222-227.

Johnston J, B Wheeler, J McKinlay (1999) Forage production from spring cereals and cereal-pea mixtures. In: Ministry of Agriculture, Food and Rural Affairs. No. 120. Ontario, Can. Disponible en: www.omafra.gov.on.ca/english/products/fieldcrops. html\#forage (Julio 2012).

Jones O R, R N Clark (1987) Effects of furrow dikes on water conservation and dryland crop yields. Soil Sci. Soc. Amer. J. 51:13071314.

Khorasani G R, P E Bedel, J H Helm, J J Kennelly (1997) Influence of stage of maturity on yield components and chemical composition of cereal grain silages. Can. J. Anim. Sci. 77:259-267.

Moore J E, D J Undersander (2002) Relative forage quality: an alternative to relative feed value and quality index. In: 13th Annual Florida Ruminant Nutrition Symp., Florida Dairy Extension (ed.). University of Florida. 10-11 Jan. pp:16-32.

NRC, National Research Council (2001) Nutrient Requirements of Dairy
Cattle. 7th ed. National Academy Press. Washinton, D. C. $408 \mathrm{p}$

Oba M, M S Allen (1999) Evaluation of the importance of the digestibility of neutral detergent fiber from forage: effects on dry matter intake and milk yield of dairy cows. J. Dairy Sci. 82:589-596.

Ortiz H (2004) Furrow dyking for soil and water conservation: Mexico, sharing innovative experiences. In: Examples of the Successful Conservation and Sustainable Use of Dryland Biodiversity. United Nations Development Programme, Vol. 9, 127 p.

Ramírez L M R, M R Ávila M (1996) El agroecosistema temporalero de la baja Babícora y regiones similares: su estructura actual, algunos de sus biocomponentes y su diseño futuro. Folleto Científico No. 3. CESICH-CIRNOC-INIFAP-SAGARPA. Ciudad Cuauhtémoc, Chihuahua, México. 46 p.

Rosser C L, P Górka, A D Beattie, H C Block, J J McKinnon, H A Lardner, G B Penner (2013) Effect of maturity at harvest on yield chemical composition, and in situ degradability for annual cereals used for swath grazing. J. Anim. Sci. 91:3815-3826.

Salmerón Z J J (2000) Teporaca, Menonita y Bachíniva, nuevas variedades de avena para el noroeste de Chihuahua. Folleto Técnico No. 12. CESICH-CIRNOC-INIFAP-SAGARPA. Ciudad Cuauhtémoc, Chihuahua, México. 18 p.

Salmerón Z J J, F J Meda, J R Barcena (2003) Variedades de avena y calidad nutricional del forraje. Folleto Técnico No. 17. CESICHCIRNOC-INIFAP-SAGARPA. Ciudad Cuauhtémoc, Chihuahua, México. 43 p.

SAS Institute (2004) SAS/STAT ${ }^{\circledast} 9.1$ User's Guide. SAS Inst. Inc., Cary, NC.

SIACON, Sistema de Información Agroalimentaria de Consulta. Servicio de Información Agroalimentaria y Pesquera (SIAP), SAGARPA, México. Disponible en: www.sagarpa.gob.mx. (Julio 2011).

Undersander D D, D R Mertens, N Thiex (1993) Forage Analysis Procedures. National Forage Testing Association Proceeding, Omaha, NE. 118 p.

Van Soest P J, J B Robertson, B A Lewis (1991) Methods for dietary fiber, neutral detergent fiber, and non-starch polysacharides in relation to animal nutrition. J. Dairy Sci. 74:3583-3589.

Wallsten J, E Nadeau, J Bertilsson, K Martinsson (2009) Voluntary intake and diet selection by dairy heifers fed ensiled whole-crop barley and oats harvested at different stages of maturity. Livestock Sci. 122:94-98. 